\title{
DETERMINAÇÃO DA OPERAÇÃO DE UNIDADES TÉRMICAS PARA O ESTUDO DE UNIT COMMITMENT ATRAVÉS DE UMA ANÁLISE DE SENSIBILIDADE
}

\author{
Ivo Chaves da Silva Junior* \\ ivojeufrj.br \\ Edimar José de Oliveira ${ }^{\dagger}$ \\ edimar@lacee.ufjf.br \\ Paulo Augusto N. Garcia ${ }^{\dagger}$ \\ pgarciallacee.ufjf.br
}

\author{
Sandoval Carneiro Junior* \\ sandovaledee.ufrj.br
}

José Luiz Rezende Pereira ${ }^{\dagger}$

jluiz@ieee.org

André L.M.Marcato ${ }^{\dagger}$

andre.marcato@ufjf.edu.br

*Universidade Federal do Rio de Janeiro-RJ

${ }^{\dagger}$ Universidade Federal de Juiz de Fora-MG

\section{RESUMO}

Este artigo propõe a utilização de um novo índice de sensibilidade à elaboração de uma lista de prioridade para a resolução do problema de "Unit Commitment". O novo índice é obtido através dos multiplicadores de Lagrange associado às variáveis discretas inerentes ao problema, as quais são mitigadas através de uma função contínua. Desta forma, o problema de otimização não linear é resolvido através da utilização da metodologia primal-dual de pontos interiores e como subproduto têm-se os multiplicadores de Lagrange associados às variáveis de decisão “ $O N-O F F$ ” para a elaboração de uma lista de prioridade. Os resultados obtidos mostram a eficiência da metodologia proposta.

PALAVRAS-CHAVE: Multiplicadores de Lagrange, função sigmoidal, pontos interiores, lista de prioridade, operação de unidades térmicas.

\footnotetext{
Artigo submetido em 30/05/2006

1a. Revisão em 14/07/2006

2a. Revisão em 14/08/2006

Aceito sob recomendação do Editor Associado

Prof. José Roberto Castilho Piqueira
}

\begin{abstract}
This paper proposes the utilization of a new sensibility index to organize a priority list for the solution of the Unit Commitment problem. The discrete nature of the operational decisions is mitigated through a continuous function. Thus the new index is derived from the lagrangian multipliers and the non-linear optimization problem is solved using the interior point primal-dual method. The priority list is obtained as a by-product of the lagrangian multipliers associated with the "ON-OFF" decision. The efficiency of the proposed methodology is demonstrated through several tests.
\end{abstract}

KEYWORDS: Lagrange coefficients, sigmoid function, interior-point, list of priority, unit commitment.

\section{INTRODUÇÃO}

A demanda de energia elétrica e a atividade humana seguem ritmos semelhantes, sendo estes ditados pela sociedade através de suas componentes residenciais, comerciais e industriais. Diante destes ritmos, que crescem de forma acentuada, o atendimento a demanda de forma econômica torna-se um fator importante na operação dos sistemas elétricos de potência. 
$\mathrm{O}$ atendimento econômico a demanda, conhecida também como despacho econômico (DE), visa determinar unicamente a potência de saída de cada unidade geradora do sistema de forma a minimizar o custo total de operação. Entretanto, o DE não considera a variação da demanda ao longo do tempo. (Srinivasan and Chazelas, 2004).

Esta análise é importante, pois possibilita o desligamento e/ou acionamento de determinadas unidades geradoras durante um determinado período de operação pré-estabelecido, o que envolve novos custos e restrições técnicas que limitam as opções de decisão (Sasaki et. al., 2004).

Desta forma, a seleção das unidades geradoras, a serem colocadas em serviço, representa um problema hierarquicamente superior ao despacho econômico. Este problema também é resolvido através de técnicas de otimização e designado como "Unit Commitment" (Narayana, 2004).

O "Unit Commitment" é formulado como um problema de programação não linear inteira mista que apresenta algumas particularidades, tais como: (i) Região de Solução não Convexa, o que permite a existência de várias soluções e conduz grande parte dos algoritmos a convergirem em direção de mínimos locais (Viana et. al., 2003); (ii) Natureza Combinatória do processo de Decisão, que leva ao fenômeno da explosão combinatória referente às alternativas de geração, acarretando elevado tempo computacional (Valenzuela and Smith, 1999); (iii) Natureza Dinâmica do processo de Decisão, que limita as opções de decisão e conseqüentemente ocasiona antagonismo em relação ao despacho econômico (Yang et. al., 1996). As dificuldades descritas anteriormente evidenciam que o desenvolvimento de técnicas e algoritmos que conciliem rapidez, robustez e eficiência na resolução do problema de "Unit Commitment" é um campo atrativo de estudo e pesquisa.

Entre as principais técnicas utilizadas na resolução do problema pode-se mencionar: lista de prioridade (Lee, 1991; Lee and Feng, 1992), programação dinâmica (Lowery, 1996; Pang et. al., 1981), programação inteira (Lauer et. al.,1981; Huang et. al., 1998) e relaxação Lagrangeana (Cheng et. al., 2000; Ongsakul and Petcharaks, 2004; Zhuang and Galiana, 1988; Aoki et. al., 1987). Meta-heurísticas como algoritmo genético (Swarup and Yamashiro, 2002; Damousis et. al., 2004) "simulated annealing" (Mantawy et. al., 1998; Simopoulos et. al., 2006), busca tabu (Rajan et. al., 2002) e "ant colony" (Sum and Ongsakul, 2003) também têm sido bastante utilizadas, além de técnicas híbridas (Srinivasan and Chazelas, 2003; Huang and Huang, 1997). Entretanto, apesar das inúmeras técnicas utilizadas a solução mínima global só pode ser garantida através da enumeração completa de todas as alternativas de operação, o que normalmente é inviável.
Considerando a inviabilidade prática da obtenção de todas as enumerações possíveis foi proposta inicialmente por (Burns and Gibson, 1975) a técnica baseada em uma lista de prioridade. Esta técnica consiste em colocar em serviço as diversas unidades geradoras de acordo com uma determinada ordem de mérito, a qual geralmente baseia-se em características econômicas e/ou grandezas relacionadas com a eficiência energética de cada unidade geradora. Desta forma uma ordem é pré-determinada e então as unidades são colocadas em serviço de modo que as restrições do problema sejam satisfeitas. Estes algoritmos têm a vantagem da simplicidade e do baixo esforço computacional. Entretanto, normalmente, conduzem a soluções de alto custo de operação.

Este artigo propõe a utilização de um novo índice de sensibilidade para a elaboração de uma lista de prioridade para a resolução do problema de "Unit Commitment". Este novo índice de sensibilidade é obtido através dos multiplicadores de Lagrange associados as variáveis discretas de decisão, as quais são mitigadas através de uma função sigmoidal. $\mathrm{O}$ problema de otimização é resolvido através da utilização da metodologia primal-dual de pontos interiores (Quintana $\boldsymbol{e t}$.

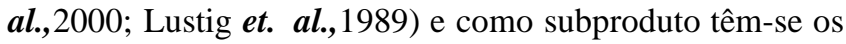
multiplicadores de Lagrange associados as variáveis de decisão para a elaboração de uma lista de prioridade. Com a lista de prioridade obtida as unidades são colocadas em serviço e procedimentos heurísticos são utilizados na elaboração da programação final de operação. Os resultados obtidos, através da simulação de um sistema teste com 4 unidades e de sistemas com 10, 20, 40, 60, 80 e 100 unidades geradoras, apontam para uma satisfatória eficiência da metodologia proposta.

\section{FORMULAÇÃO PROPOSTA}

\subsection{Notação utilizada:}

$N$ Número total de unidades térmicas;

$T$ Período total de operação;

$i$ Índice da unidade térmica;

$t$ Índice da hora;

$O D_{i}\left(x_{i}^{t}\right)$ Variável discreta [0,1] de decisão "ON/OFF", mitigada pela função sigmoidal;

$P_{i}(t)$ Potência ativa gerada pela unidade térmica $i$ na hora $t$;

$P_{i}^{\max }$ Limite máximo de potência ativa gerada pela unidade térmica $i$;

$P_{i}^{\text {min }}$ Limite mínimo de potência ativa gerada pela unidade térmica $i$;

$D(t)$ Demanda solicitada na hora $t$; 
$r(t)$ Reserva girante solicitada na hora $t$;

$T_{i}^{o n}$ Tempo mínimo de partida da unidade térmica $i$;

$T_{i}^{o f f}$ Tempo mínimo de parada da unidade térmica $i$;

$X_{i}^{o n}(t)$ Tempo em que a unidade térmica $i$ está ligada;

$X_{i}^{o f f}(t)$ Tempo em que a unidade térmica $i$ está desligada;

$S C_{i}(t)$ Custo de partida da unidade térmica $i$ na hora $t$;

$S D_{i}(t)$ Custo de parada da unidade térmica $i$ na hora $t$;

$a_{i}, b_{i}, c_{i}$ Coeficientes referentes aos custos do combustível da unidade térmica $i\left(\$ / \mathrm{h}, \$ / \mathrm{MWh}, \$ / \mathrm{MW}^{2} \mathrm{~h}\right)$;

$h c_{i}$ Custo de partida quente da unidade térmica $i$;

$c c_{i}$ Custo de partida fria da unidade térmica $i$;

$c s h_{i}$ Tempo de partida fria da unidade térmica $i$;

$\left.P_{C}^{(} t\right)$ Potência ativa gerada pela unidade de convergência na hora $t$

$P_{C}^{\max }(t)$ Limite máximo de potência ativa gerada pela unidade de convergência;

$P_{C}^{\min }(t)$ Limite mínimo de potência ativa gerada pela unidade convergência;

$\rho$ Custo da unidade de convergência (alto custo);

$x_{i}^{t}$ Argumento da função sigmoidal da unidade térmica ina hora $t$;

$x^{\min }, x^{\max }$ Limite mínimo e máximo do argumento da função sigmoidal;

$\pi_{x i}(t)$ Multiplicador de Lagrange associado ao argumento da função sigmoidal no instante $t$;

$\pi_{p}(t)$ Multiplicador de Lagrange associado ao limites de potência ativa no instante $t$;

$\lambda_{d}(t)$ Multiplicador de Lagrange associado à equação de balanço de potência no instante $t$;

$\lambda_{r}(t)$ Multiplicador de Lagrange associado à restrição de reserva girante no instante $t$;

$C I_{i}$ Condição inicial da unidade geradora $i$; $\mathrm{O}$ sinal (+) representa “ON" e (-) representa “OFF”.

1) Função Objetivo: A função objetivo (FOB), equação (1), consiste na minimização da soma do custo total de operação (A) e dos custos de partida (B) e parada (C) das unidades geradoras durante o período de operação em estudo. Uma parcela adicional (D) é incluída na FOB tradicional do problema com objetivo de possibilitar a convergência e a obtenção dos multiplicadores de Lagrange (ML) associados às variáveis de decisão $[0,1]$ das unidades geradoras. A obtenção destes multiplicadores é abordada no apêndice A.

$$
\text { Min } \mathrm{FOB}=\mathrm{A}+\mathrm{B}+\mathrm{C}+\mathrm{D}
$$

onde:

$$
\begin{gathered}
\mathrm{A}=\sum_{\mathrm{t}=1}^{\mathrm{T}} \sum_{i=1}^{N}\left[a_{i}+b_{i} \cdot P_{i}(t)+c_{i} \cdot P_{i}^{2}(t)\right] \cdot O D_{i}\left(x_{i}^{t}\right) \\
B=\sum_{\mathrm{t}=1}^{\mathrm{T}} \sum_{i=1}^{N} S C_{i}(t) \\
\mathrm{C}=\sum_{\mathrm{t}=1}^{\mathrm{T}} \sum_{i=1}^{N} S D_{i}(t) \\
\mathrm{D}=\sum_{t=1}^{T} \rho \cdot P_{C}(t)
\end{gathered}
$$

É importante mencionar que o custo de partida ou arranque das unidades geradoras depende do tempo que a unidade esteve parada anteriormente e do fato de se manter ou não as caldeiras quentes durante o período de parada. Desta forma, o custo de partida pode ser definido pela equação (6).

$$
\mathrm{SC}_{\mathbf{i}}(t)=\left\{\begin{array}{c}
h c_{i}: T_{i}^{o f f} \leq X_{i}^{o f f}(t) \leq T_{i}^{o f f}+c s h_{i} \\
c c_{i}: X_{i}^{o f f}>T_{i}^{o f f}+c s h_{i}
\end{array}\right\}
$$

O custo de parada (SD) é sempre dado como um valor constante para cada unidade. Neste artigo adotou-se custo de parada igual a zero para todas as unidades.

2) Restrição de Balanço de Potência: A restrição de balanço de potência ativa, equação (7), analisa diretamente, através da primeira lei de Kirchhoff, o estado de equilíbrio do sistema a todo instante de tempo.

$$
\sum_{i=1}^{N} O D_{i}\left(x_{i}^{t}\right) \cdot P_{i}(t)=D(t) \ldots \lambda_{\mathrm{d}}(t)
$$

3) Restrição de Reserva Girante: É necessário prever uma folga, designada de reserva girante, entre a carga prevista e a potência total disponível entre as unidades geradoras em 
serviço para suprir aumentos inesperados de carga ou desvios de previsão. A equação (8) traduz esta folga.

$$
\sum_{i=1}^{N} O D_{i}\left(x_{i}^{t}\right) \cdot P_{i}^{\max } \geq D(t)+r(t) \ldots \lambda_{\mathbf{r}}(t)
$$

4) Restrições das Unidades Geradoras: Em relação às unidades geradoras foram consideradas as seguintes restrições: (i) tempos mínimos de partida e parada, equações (9) e (10) respectivamente; (ii) limites máximos e mínimos de produção, equação (11).

$$
\begin{gathered}
X_{i}^{o n}(t) \geq T_{i}^{o n} \\
X_{i}^{o f f}(t) \geq T_{i}^{o f f} \\
P_{i}^{\min } \leq P_{i}(t) \leq P_{i}^{\max } \ldots \pi_{\mathrm{pi}}(t)
\end{gathered}
$$

\section{5) Representação dos Estados “ON-OFF” das Unidades}

Geradoras: De modo a evitar as dificuldades peculiares da resolução de problemas de natureza discreta, permitiu-se que as variáveis de decisão assumam valores contínuos dentro do intervalo discreto $[0,1]$; esta estratégia enseja a obtenção dos coeficientes de Lagrange associados a estas variáveis. A função sigmoidal, denominada função decisão de operação, para a representação dos estados "ON-OFF" das unidades, equação (12), foi adotada devido à similaridade com a função degrau, figura 1. A equação (13) é referente à restrição de canalização do argumento da função sigmoidal.

$$
\begin{gathered}
O D_{i}\left(x_{i}^{t}\right)=\frac{e^{\alpha x_{i}^{t}}-1}{e^{\alpha x_{i}^{t}}+1} \\
x_{i}^{\min } \leq x_{i}^{t} \leq x_{i}^{\max } \ldots \pi_{\mathbf{x i}}(t)
\end{gathered}
$$

Assim como em (Oliveira et. al., 2005), vários valores para o parâmetro $\alpha$ foram testados e pôde-se verificar que o valor unitário representa um bom compromisso entre convergência e qualidade dos resultados.

\section{METODOLOGIA PROPOSTA}

A metodologia proposta pode ser divida em quatro etapas: (i) Obtenção dos índices de sensibilidade; (ii) Colocação das unidades em serviço; (iii) Tratamento das restrições referentes aos tempos mínimos de partida e parada das unidades geradoras; (iv) Despacho econômico. O fluxograma do algoritmo proposto é apresentado pela figura 2 e a seguir são descritas as etapas utilizadas pela metodologia proposta para o estudo de "Unit Commitment"

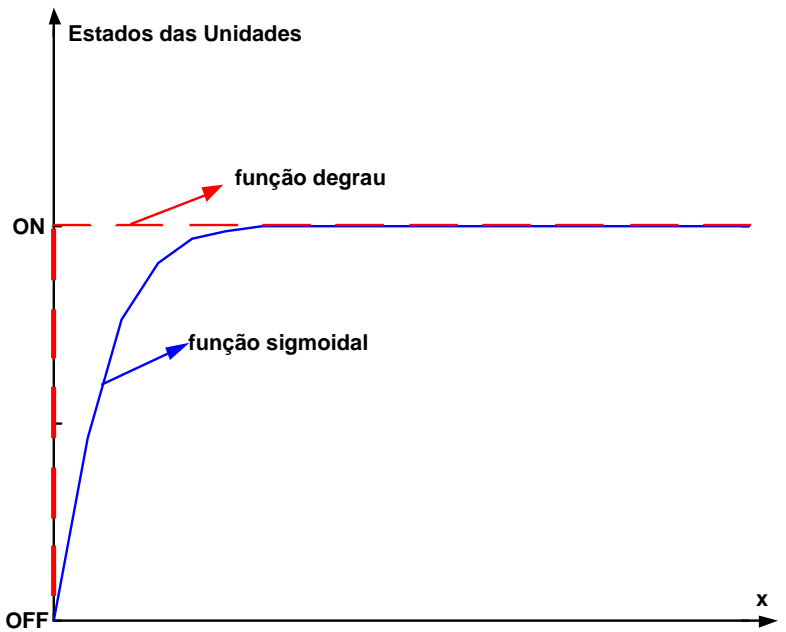

Figura 1: Função Decisão de Operação.<smiles>[AlH2]</smiles>

Obtenção dos Índices de Sensibilidade

(2)

Geração do Plano Parcial de Operação

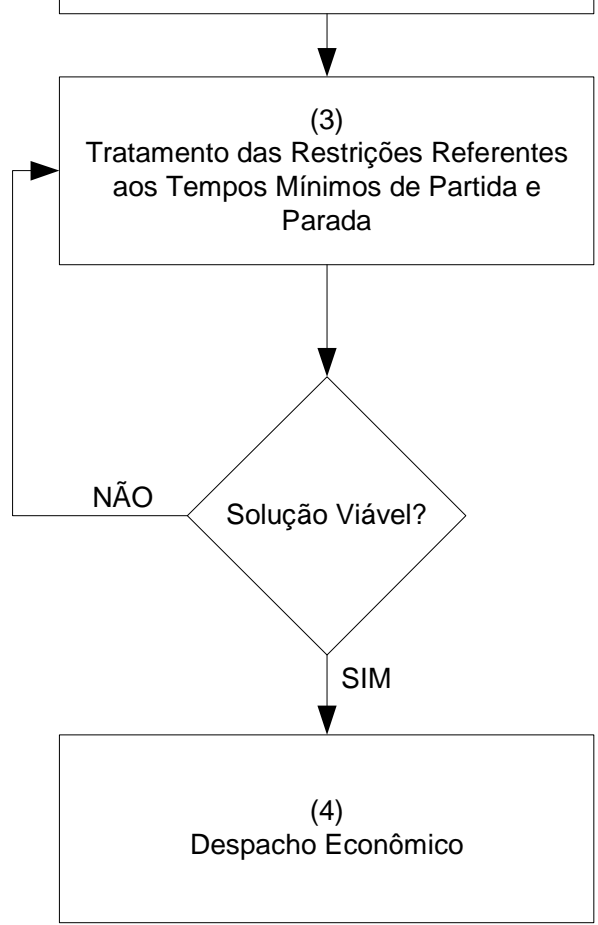

Figura 2: Fluxograma do Algoritmo Proposto. 
1) Obtenção dos Índices de Sensibilidade:Considerando a já mencionada inviabilidade de obtenção de todas as enumerações possíveis, a qual constituí a única forma de se garantir o ponto de mínimo global, a metodologia proposta utiliza um novo índice de sensibilidade com o objetivo de identificar as unidades geradoras de maior relevância técnica e econômica. O novo índice é obtido através dos seguintes passos:

(i) Adota-se um intervalo estreito de canalização para o argumento da função de decisão, ou seja, limites máximos e mínimos das variáveis $x_{i}^{t}$ próximos de zero, uma vez que se pretende avaliar a sensibilidade do acionamento de cada uma das unidades geradoras. Este estreitamento tem como objetivo a obtenção dos coeficientes de Lagrange associados à função de decisão de cada unidade geradora $i$ na horat. Assim, adotou-se $0 \leq x_{i}^{t} \leq 0,0001$.

(ii) Adiciona-se uma unidade geradora denominada gerador de convergência na análise. Este gerador possui como característica alto custo de geração em relação ao custo das demais unidades e tem por objetivo fazer com que o fluxo de potência ótimo "FPO" tenha solução. Portanto, nesta etapa, cabe a este gerador suprir sozinho a demanda e a reserva girante do sistema. Para as demais etapas, esta unidade não é considerada.

(iii) A seguir, resolve-se o FPO para cada hora até o fim do período de operação $T$ previamente conhecido. Ao final, obtém-se uma matriz de sensibilidade (SM), equação (14). Esta matriz é formada pelos multiplicadores de Lagrange associados ao argumento da função de decisão, equação (13), para cada uma das unidades geradoras durante todo o período de operação.

$$
S M=\left[\begin{array}{cccc}
\pi_{x 1}(t) & \pi_{x 2}(t) & \ldots & \pi_{x N}(t) \\
\pi_{x 1}(t+1) & \pi_{x 2}(t+1) & \ldots & \pi_{x N}(t+1) \\
: & : & \ldots & : \\
\pi_{x 1}(T) & \pi_{x 2}(T) & \ldots & \pi_{x N}(T)
\end{array}\right]
$$

A matriz de sensibilidade $(S M)$ obtida retrata a sensibilidade da função objetivo em relação à variação da função de decisão de cada uma das unidades geradoras ao longo do período de operação. Entretanto, como estes multiplicadores são de caráter local (horário), optou-se por calcular a média destes multiplicadores de Lagrange para cada uma das unidades, equação (15), com o objetivo de se obter uma sensibilidade geral e com isso uma ordem de mérito.

$$
\bar{\pi}_{x i}=\frac{\sum_{t=1}^{T} \pi_{x i}(t)}{T} \mathrm{i}=1, \ldots ., \mathrm{N}
$$

Como os valores dos multiplicadores são negativos, já que estes retratam a redução da função objetivo em relação à tendência da colocação das unidades em serviço, a ordem de mérito é obtida através da ordenação crescente dos valores médios dos multiplicadores de Lagrange.

2) Colocação das Unidades Geradoras em Serviço: Com a ordem de mérito obtida as unidades geradoras são colocadas em serviço de modo que as equações (16) e (17) sejam satisfeitas, pois estas restrições garantem o atendimento à demanda.

$$
\begin{gathered}
\sum P^{\min }(t) \leq D(t) \\
\sum P^{\max }(t) \geq D(t)+r(t)
\end{gathered}
$$

Entretanto, verificou-se que há determinados trechos da curva de carga que merecem atenção especial na colocação das unidades em serviço. Estes trechos correspondem aos pontos de demanda mínima e valores próximos a este. Isto se deve principalmente quando a unidade mais econômica não consegue atender sozinha a restrição (17) no ponto mínimo da curva de carga. Nesta situação são necessários apenas pequenos blocos de energia, por um período curto de tempo, para complementar a geração já disponível e/ou a reserva girante solicitada.

Para lidar com este problema, optou-se por classificar dois tipos de unidades: (i) Unidade permanente - unidade escolhida para permanecer em serviço durante todo período de operação, sendo esta a primeira da lista de ordem de mérito, ou seja, a mais econômica; (ii) Unidade de apoio - unidade que tem como objetivo, se possível, complementar a geração da unidade permanente e /ou a reserva girante no ponto de demanda mínima ou pontos próximos a este. Esta unidade deve ter como característica principal alta flexibilidade de operação, ou seja, tempos mínimos de parada e partida curtos. Apesar de unidades com estas características possuírem, normalmente, custos de operação mais onerosos, a flexibilidade de operação e seu baixo custo de partida tornam estas unidades relevantes no suprimento de pequenos blocos de energia. È importante mencionar que se houver mais de uma unidade com as características mencionadas, seleciona-se a unidade de apoio pela ordem de mérito estabelecida. Para os demais pontos de demanda, a ordem de mérito é que dita os estados de operação das unidades geradoras, independente de suas características técnicas e/ou econômicas. 
Desta forma, um plano de operação parcial é obtido e partese para a etapa referente ao tratamento dos tempos mínimos de parada e partida das unidades geradoras, uma vez que estes não foram considerados na obtenção do plano parcial de operação.

\section{3) Tratamento dos Tempos Mínimos de Partida e Parada das Unidades Geradoras:}

Sabe-se que o comportamento da demanda durante o período de operação é o fator determinante para a definição das transições dos estados possíveis "ON-OFF" para cada uma das unidades geradoras. Assim, sem uma visão dinâmica do comportamento da demanda, as decisões de acionamento ou desligamento das unidades podem ser equivocadas. Diante disso, optou-se por fazer a análise dos tempos mínimos das unidades somente depois da obtenção do plano de operação parcial, já que através deste se tem um panorama geral de cada unidade geradora mediante o comportamento da demanda ao longo de todo período de operação.

Desta forma, com base em (Senjyu et. al., 2003), a seguinte análise heurística é proposta: $(i)$ identificação das unidades que tiveram violações em suas restrições de tempo mínimo de parada e/ou partida; (ii) tratamento das transições " $O F F$ $O N$ ”, ou seja, se o tempo de operação da unidade $i$, dentro do período mínimo de funcionamento, for maior ou igual a $2 / 3$ de $T_{i}^{o n}$, liga-se a unidade. Caso contrário, mantém-se a unidade desligada; (iii) o tratamento das transições "ON-OFF" é feito de forma análoga, ou seja, se o tempo de desligamento da unidade $i$, dentro do período mínimo de parada, for maior ou igual a $2 / 3$ de $T_{i}^{o f f}$, desliga-se a unidade. Caso contrário mantém-se a unidade ligada.

Com isso, modificações no plano parcial de operação e os seguintes cenários não desejáveis podem ocorrer: $(i)$ excessos de reserva girante, os quais podem ser amenizados desligando as unidades de pior classificação, desde que este desligamento não acarrete violação da restrição de tempo mínimo de parada da unidade selecionada; (ii) não atendimento a demanda e/ou reserva que pode ser corrigido ligando as unidades de melhor classificação entre as desligadas, desde que este acionamento também não acarrete violação da restrição de tempo mínimo de partida da unidade selecionada.

Os procedimentos descritos acima devem ser realizados até a obtenção de uma solução totalmente viável, ou seja, que respeite as restrições de reserva girante, demanda, tempos mínimos de parada e tempos mínimos de partida. Desta forma, tem-se um plano final de operação.

4) Despacho Econômico: Com a definição final dos estados "ON-OFF" das unidades geradoras durante todo o período de operação parte-se para o problema referente ao despacho econômico. Assim, a metodologia proposta utiliza

\begin{tabular}{|c|c|c|c|c|}
\hline Unidades & UTE 1 & UTE 2 & UTE 3 & UTE 4 \\
\hline$P_{i}^{\max }$ & 300 & 250 & 80 & 60 \\
\hline$P_{i}^{\min }$ & 75 & 60 & 25 & 20 \\
\hline$a_{i}$ & 684.74 & 585.62 & 213.00 & 252.00 \\
\hline$b_{i}$ & 16.83 & 16.95 & 20.74 & 23.60 \\
\hline$c_{i}$ & 0.0021 & 0.0042 & 0.0018 & 0.0034 \\
\hline$T_{i}^{\text {on }}$ & 5 & 5 & 4 & 1 \\
\hline$T_{i}^{\text {off }}$ & 4 & 3 & 2 & 1 \\
\hline$h c_{i}$ & 500 & 170 & 150 & 0.00 \\
\hline$c c_{i}$ & 1100 & 400 & 350 & 0.02 \\
\hline$c s h_{i}$ & 5 & 5 & 4 & 0 \\
\hline$C I_{i}$ & +8 & +8 & -5 & -6 \\
\hline
\end{tabular}

Tabela 1: Características das Unidades-Sistema Teste.

\begin{tabular}{|c|c|c|c|c|c|}
\hline$t$ & $D(t)$ & $r(t)$ & $t$ & $D(t)$ & $r(t)$ \\
\hline 1 & 450 & 45 & 5 & 400 & 40 \\
\hline 2 & 530 & 53 & 6 & 280 & 28 \\
\hline 3 & 600 & 60 & 7 & 290 & 29 \\
\hline 4 & 540 & 54 & 8 & 500 & 50 \\
\hline
\end{tabular}

Tabela 2: Demanda e Reserva Girante-Sistema Teste.

novamente o FPO baseado no método de pontos interiores (Wright, 1997) para a resolução do despacho econômico.

\section{ESTUDO DE CASOS}

Com o objetivo de verificar a eficiência da metodologia proposta foram realizadas simulações através de um sistema tutorial com quatro unidades térmicas (UTE) (Valenzuela and Smith, 1999) e sistemas com 10, 20, 40, 60, 80 e 100 unidades (Senjyu et. al., 2003). Os dados referentes ao sistema tutorial são apresentados nas tabelas 1 e 2. Os dados referentes aos demais sistemas estão ilustrados no apêndice B.

\subsection{Sistema Tutorial com 4 Unidades Tér- micas}

A figura 3 apresenta o diagrama unifilar do sistema teste pela modelagem proposta, onde são representadas as unidades térmicas e suas respectivas funções de decisão, representando os estados "ON-OFF" inerentes ao problema. Como já mencionado, a inclusão de uma unidade extra, denominada de unidade de convergência, se faz necessária a fim de possibilitar a convergência do FPO. Convergência esta que não seria 


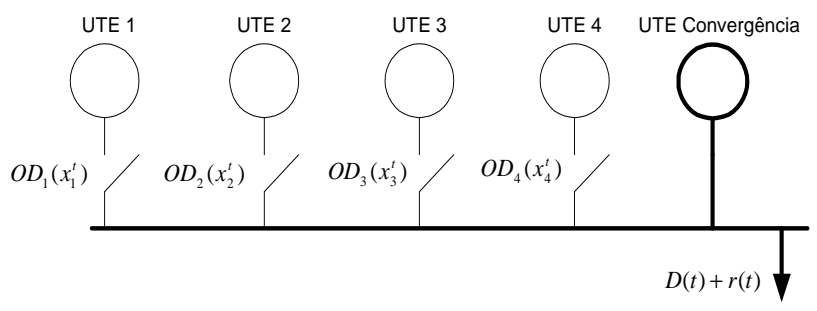

Figura 3: Diagrama Unifilar-Sistema Teste.

\begin{tabular}{|c|c|c|c|c|}
\hline$t$ & $\pi_{x 1}(t) *$ & $\pi_{x 2}(t) *$ & $\pi_{x 3}(t) *$ & $\pi_{x 4}(t) *$ \\
\hline 1 & -2.6208 & -2.1764 & -0.6821 & -0.4652 \\
\hline 2 & -2.3163 & -1.9226 & -0.6009 & -0.4043 \\
\hline 3 & -1.0693 & -0.8834 & -0.2684 & -0.1549 \\
\hline 4 & -2.2831 & -1.8949 & -0.5921 & -0.3976 \\
\hline 5 & -2.7645 & -2.2961 & -0.7204 & -0.4939 \\
\hline 6 & -4.5429 & -3.7781 & -1.1946 & -0.8495 \\
\hline 7 & -4.4669 & -3.7147 & -1.1744 & -0.8343 \\
\hline 8 & -2.4301 & -2.0174 & -0.6313 & -0.4270 \\
\hline
\end{tabular}

Tabela 3: Matriz de Sensibilidade - Sistema Teste $\left(* 10^{3}\right.$.)

obtida devido ao estreitamento das restrições de canalização do argumento da função de operação, equação (13).

É importante ressaltar que a unidade de convergência tem como característica alto valor de custo quando comparado com os custos das demais unidades. Desta forma, o FPO irá inicialmente tentar colocar as unidades geradoras mais econômicas em operação, porém com a imposição feita às restrições de canalização de $O D(x)$, as restrições (16) e (17) só podem ser garantidas pela utilização da unidade de convergência. Desta forma, tem-se como produto do FPO os multiplicadores de Lagrange associados aos argumentos da função de decisão de cada uma das unidades geradoras.

O FPO deverá ser calculado para todo o período de operação, 8 horas. A tabela 3 apresenta os elementos da matriz de sensibilidade correspondente aos multiplicadores de Lagrange associados à função de decisão de cada uma das quatro unidades geradoras para todo o período de operação. $\mathrm{O}$ sinal negativo representa a redução na função objetivo associada à tendência de acionamento da unidade térmica, ou seja, $O D_{i}\left(x_{i}^{t}\right) \rightarrow 1$.

Entretanto, como estes multiplicadores são de caráter local, optou-se por calcular a média destes multiplicadores de Lagrange (ML) para cada uma das unidades com o objetivo de se obter uma sensibilidade geral e com isso uma ordem de mérito. A tabela 4 apresenta os valores médios obtidos, através da equação (15), e a ordem de mérito estabelecida.

\begin{tabular}{|c|c|c|c|c|}
\hline & UTE 1 & UTE 2 & UTE 3 & UTE 4 \\
\hline $\begin{array}{c}\text { Média dos } \\
\text { ML }\end{array}$ & -2.811 & -2.335 & -0.733 & -0.5033 \\
\hline $\begin{array}{c}\text { Ordem de } \\
\text { Mérito }\end{array}$ & $1^{a} \cdot$ & $2^{a}$. & $3^{a}$. & $4^{a}$. \\
\hline
\end{tabular}

Tabela 4: Ordem de Mérito - Sistema Teste.

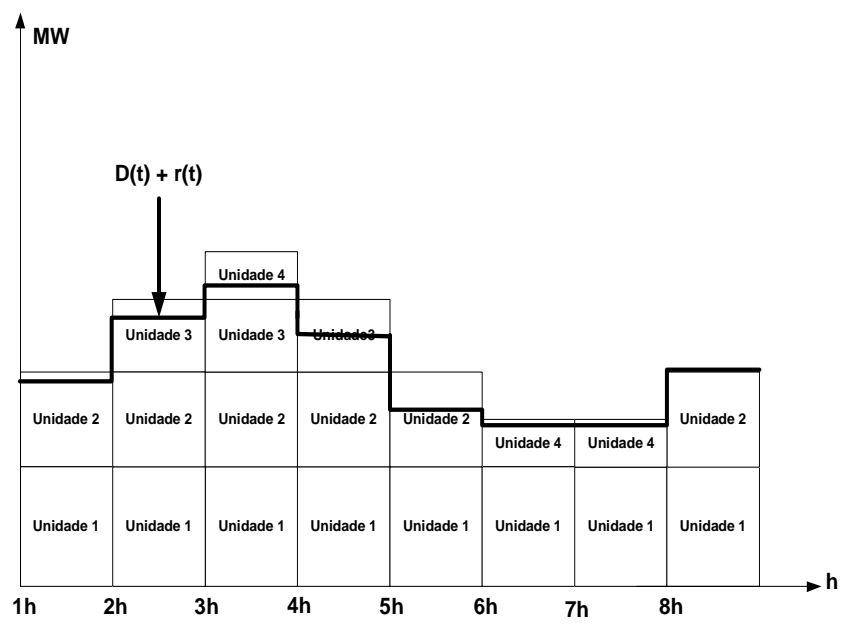

Figura 4: Plano Parcial de Operação-Sistema Teste.

Através da ordem de mérito obtida, pode-se definir a unidade permanente. A unidade permanente corresponde à unidade mais econômica, sendo esta a primeira unidade da ordem de mérito, e, portanto a escolhida para permanecer em serviço por todo período de operação. Já a unidade de apoio, unidade quatro, corresponde à térmica de menor tempo de partida entre as unidades. Isto por que esta unidade tem por objetivo somente complementar a geração da unidade permanente e/ou reserva girante requerida nas condições mínimas de carregamento.

A seguir, as unidades são colocadas em serviço de acordo com a ordem de mérito obtida de modo que as equações (16) e (17) sejam satisfeitas. Deve-se sempre verificar a possibilidade da utilização da unidade de apoio nos pontos de demanda mínima (6h) ou pontos de carga com valores próximos a este (7h). Com isso, obtém-se um plano parcial de operação para o sistema teste, apresentado pela figura 4.

Analisando a figura 4 é possível verificar que a unidade 2 e 3 violam seus respectivos tempos mínimos de parada e partida. A unidade 3 foi colocada em serviço às 2 horas e deveria permanecer em serviço, no mínimo, até as 6 horas. Entretanto, pode-se verificar que a unidade foi desligada às 5 ho- 


\begin{tabular}{|c|c|c|c|c|}
\hline$t$ & UTE 1 & UTE 2 & UTE 3 & UTE 4 \\
\hline 1 & ON & ON & OFF & OFF \\
\hline 2 & $O N$ & ON & ON & OFF \\
\hline 3 & ON & ON & ON & ON \\
\hline 4 & ON & ON & ON & OFF \\
\hline 5 & ON & OFF & ON & ON \\
\hline 6 & ON & OFF & OFF & ON \\
\hline 7 & ON & OFF & OFF & ON \\
\hline 8 & ON & ON & OFF & OFF \\
\hline
\end{tabular}

Tabela 5: Plano Final de Operação -Sistema Teste.

\begin{tabular}{|c|c|c|c|c|}
\hline$t$ & $\begin{array}{c}\text { UTE 1 } \\
(\mathrm{MW})\end{array}$ & $\begin{array}{c}\text { UTE 2 } \\
\text { (MW) }\end{array}$ & $\begin{array}{c}\text { UTE 3 } \\
(\mathrm{MW})\end{array}$ & $\begin{array}{c}\text { UTE 4 } \\
(\mathrm{MW})\end{array}$ \\
\hline 1 & 300 & 150 & 0 & 0 \\
\hline 2 & 300 & 205 & 25 & 0 \\
\hline 3 & 300 & 250 & 30 & 20 \\
\hline 4 & 300 & 215 & 25 & 0 \\
\hline 5 & 300 & 0 & 80 & 20 \\
\hline 6 & 260 & 0 & 0 & 20 \\
\hline 7 & 270 & 0 & 0 & 20 \\
\hline 8 & 300 & 200 & 0 & 0 \\
\hline
\end{tabular}

Tabela 6: Despacho Econômico -Sistema Teste.

ras, violando a restrição referente ao tempo mínimo de partida da unidade, veja tabela 1. Fazendo a análise heurística proposta, dentro do período mínimo de funcionamento (2h$6 \mathrm{~h})$, verificou-se que a unidade permaneceu ligada por um período superior a $2 / 3$ do tempo mínimo de partida, sendo assim, a unidade deve ser mantida em serviço até às $6 \mathrm{~h}$. Para a unidade 2 o procedimento análogo é realizado e o plano de operação final é obtido, conforme tabela 5.

Depois de definido quais unidades devem estar em serviço a cada hora, parte-se para a última etapa, correspondente ao despacho econômico. A tabela 6 apresenta o despacho de cada uma das unidades térmicas durante todo o período de operação.

Através da tabela 6 , calcula-se o valor da função objetivo dada pela equação (1), lembrando que para esta etapa a unidade de convergência não é considerada. Desta forma, o custo de operação, dado pela equação (2) é de \$74.325,00 e o custo de partida, dada pelas equações (3) e (6), é de \$320, 00. Com isso, tem-se um custo total de $\$ 74.645,00$. Como este sistema é de pequeno porte, a enumeração total das alternati-

\begin{tabular}{|c|c|c|c|}
\hline OM & UTE & OM & UTE \\
\hline $1^{a}$ & 2 & $6^{a}$ & 6 \\
\hline $2^{a}$ & 1 & $7^{a}$ & 7 \\
\hline $3^{a}$ & 5 & $8^{a}$ & 8 \\
\hline $4^{a}$ & 4 & $9^{a}$ & 9 \\
\hline $5^{a}$ & 3 & $10^{a}$ & 10 \\
\hline
\end{tabular}

Tabela 7: Ordem de Mérito - Sistema 10 Unidades.

vas de operação é possível. O resultado desta enumeração é apresentado em (Valenzuela and Smith, 1999) e corresponde ao valor encontrado pela metodologia proposta, sendo este, portanto o ponto de mínimo global.

\subsection{Análise Comparativa de Resultados}

Os dados referentes ao sistema com 10 unidades geradoras encontram-se no apêndice B. Os dados referentes aos sistemas com 20, 40, 60, 80 e 100 unidades geradoras são obtidos duplicando o sistema base (10 unidades) e a demanda de acordo com o número de unidades. A reserva girante requerida é assumida como $10 \%$ da demanda (Senjyu et. al., 2003).

A tabela 7 apresenta a ordem de mérito (OM) obtida através da sensibilidade proposta, para o sistema base com 10 unidades geradoras. Nota-se que esta ordem retrata de maneira indireta a ordenação obtida para os demais sistemas já que estes são gerados através de duplicações do sistema base.

Os resultados encontrados pela metodologia proposta, referentes aos sistemas de 10-100 unidades, são apresentados e comparados com os últimos resultados encontrados na literatura especializada, tabelas 8 e 9. São estes: $(i)$ LR e GA (Kalazaris et. al., 1996); (ii) ELR e DPLR (Ongsakul and Petcharaks, 2004); (iii) LRGA (Cheng et. al., 2000); (iv) GAUC (Senjyu et. al., 2002); (v) EP (Juste et. al., 1999); (vi) PLEA e EPL (Srinivasan and Chazelas, 2004); (vii) ICGA (Damousis et. al.,2004).

Os valores em negrito destacam os melhores resultados encontrados para cada sistema em estudo. A última linha das tabelas (8) e (9) corresponde ao tempo de processamento referente à metodologia proposta para cada sistema em estudo. É importante ressaltar que as restrições de retomada de carga, rampa, foram negligenciadas nas simulações realizadas para permitir a comparação com os resultados obtidos por outras metodologias.

Fazendo uma análise dos resultados pode-se verificar que a metodologia proposta apresentou resultados se não melhores, 


\begin{tabular}{|c|c|c|c|}
\hline Sistemas & 10 UTE`s & 20 UTE`s & $\begin{array}{c}40 \\
\text { UTE`s }\end{array}$ \\
\hline Métodos & \multicolumn{3}{|c|}{ Custo de Produção (\$) } \\
\hline LR & 565825 & 1130660 & 2258503 \\
\hline ELR & $\mathbf{5 6 3 9 7 7}$ & 1123297 & 2244237 \\
\hline LRGA & 564800 & $\mathbf{1 1 2 2 6 2 2}$ & $\mathbf{2 2 4 2 1 7 8}$ \\
\hline DPLR & 564049 & 1128098 & 2256195 \\
\hline GA & 565825 & 1126243 & 2251911 \\
\hline GAUC & $\mathbf{5 6 3 9 7 7}$ & 1125516 & 2249715 \\
\hline EP & 564551 & 1125494 & 2249093 \\
\hline ICGA & 566404 & 1127244 & 2254123 \\
\hline PLEA & $\mathbf{5 6 3 9 7 7}$ & 1124295 & 2243913 \\
\hline EPL & $\mathbf{5 6 3 9 7 7}$ & 1124369 & 2246508 \\
\hline Proposto & $\mathbf{5 6 3 9 7 7}$ & 1123990 & 2243708 \\
\hline CPU & 10 seg & 18 seg & 27 seg \\
\hline
\end{tabular}

Tabela 8: Comparação de Resultados.

competitivos em relação a grande parte das metodologias utilizadas na resolução do "Unit Commitment". Apesar da diferença entre os resultados alcançados pela metodologia proposta e as demais serem pequenas, em alguns casos, deve-se lembrar que os custos de operação são na ordem de milhões ou até bilhões de dólares e que uma pequena redução pode ser uma cifra bastante significativa. Além disso, um aspecto importante inerente à metodologia proposta é a questão relacionada ao tempo de processamento que é substancialmente menor quando comparado com grande parte das técnicas utilizadas (Ongsakul and Petcharaks, 2004). Entretanto é importante ressaltar que nenhuma metodologia garante o ponto de mínimo global, ponto este que só é garantido através da enumeração completa das alternativas de operação.

\section{CONCLUSÃO}

Este artigo apresentou uma nova representação para a variável discreta de decisão e um novo índice de sensibilidade com o objetivo de identificar as unidades geradoras de maior relevância para o estudo de "Unit Commitment". Diante dos resultados obtidos, os seguintes aspectos podem ser enfatizados:

- Os resultados podem ser considerados promissores, uma vez que a metodologia proposta foi capaz de conciliar baixo esforço computacional com soluções de qualidade;

- Percebe-se através dos resultados que o método pro-

\begin{tabular}{|c|c|c|c|}
\hline Sistemas & 60 UTE`s & 80 UTE`s & $\begin{array}{c}100 \\
\text { UTE's }\end{array}$ \\
\hline Métodos & \multicolumn{3}{|c|}{ Custo de Produção $(\$)$} \\
\hline LR & 3394066 & 4526022 & 5657277 \\
\hline ELR & 3363491 & 4485633 & 5605678 \\
\hline LRGA & 3371079 & 4501844 & 5613127 \\
\hline DPLR & 3384293 & 4512391 & 5640488 \\
\hline GA & 3376625 & 4504933 & 5627437 \\
\hline GAUC & 3375065 & 4505614 & 5626514 \\
\hline EP & 3371611 & 4498479 & 5623885 \\
\hline ICGA & 3378108 & 4498943 & 5630838 \\
\hline PLEA & 3363892 & 4487354 & 5607904 \\
\hline EPL & 3366210 & 4489322 & 5608440 \\
\hline Proposto & $\mathbf{3 3 6 2 9 1 8}$ & $\mathbf{4 4 8 3 5 9 3}$ & $\mathbf{5 6 0 2 8 4 4}$ \\
\hline CPU & 40 seg & 54 seg & 73 seg \\
\hline
\end{tabular}

Tabela 9: Comparação de Resultados.

posto torna-se mais competitivo para sistemas de maior porte;

- A função sigmoidal mostrou-se uma aproximação eficiente para a variável discreta de decisão;

- A utilização dos multiplicadores de Lagrange associados a variável de controle da função sigmoidal mostrouse um indicador eficiente das unidades relevantes ao problema. Isto porque estes multiplicadores conseguem capturar o comportamento da função objetivo em relação à tendência de operação das usinas e o impacto das condições de carga do sistema;

- O método proposto permite a inclusão das restrições referentes à rede de transmissão e de retomada de carga das unidades geradoras (rampa);

- O algoritmo proposto também pode ser utilizado como gerador de soluções iniciais para a utilização de metaheurística, pois se sabe que eficiência do processo de busca é função das soluções iniciais factíveis.

\section{REFERÊNCIAS}

Aoki, K., Satoh, T. and Itoh, M. (1987). "Unit commitment in large scale power systems including fuel constrained thermal and pumped storage hydro," IEEE Trans. Power Syst., Vol. PWRS-2, pp. 1077-1084, May. 
Burns, R.M. and Gibson, C.A. (1975). "Optimization of Priority List for a Unit Commitment Problem", IEEE PES Summer Meeting, Paper $\mathrm{n}^{\circ}$ A75 453-1.

Cheng, C.P., Liu, C.W. and Liu, C.C. (2000). "Unit commitment by Lagrangian Relaxation and genetic algorithm," IEEE Trans. Power Syst., vol. 15, pp. 707-714, May.

Damousis, I.G.S., Bakirtziz, A.G. and Dokopoulos, P.S. (2004). "A Solution to the Unit Commitment Problem Using Integer Coded Genetic Algorithm" IEEE Trans. Power Syst., vol.19, no.2, May.

Huang, S.J. and Huang, C.L. (1997). "Application of geneticbased neural network to thermal unit commitment" IEEE Trans. Power Syst., vol. 12, pp. 654-660, May.

Huang, K.Y., Yang, H.T. and Huang, C.L. (1998). "A new thermal unit commitment approach using constraint logic programming," IEEE Trans. Power Syst., vol. 13, pp. 936-945, Aug.

Juste, K.A., Kita, H., Tanaka, E. and Hasegawa, J. (1999). "An Evolutionary Programming Solution to the Unit Commitment Problem”, IEEE Trans.Power Syst., vol.14, pp.1452-1459, Nov.

Kalazaris, S.A., Bakirtziz, A.G and Pedritis, V. (1996). “A Genetic Algorithm Solution to the Unit Commitment Problem", IEEE Trans.Power. Syst., vol.11, no.2, pp. 83-93, Feb. 1996.

Lauer,G.S., Sandell Jr, M.R., Bertsekas, N.R. and Posbergh, T.A (1982). "Solution of large scale optimal unit commitment problems," IEEE Trans. Power App. Syst., vol. PAS-101, pp. 79-96, Jan.

Lee, F.N and Feng, Q., (1992). "Multi-Area Unit Commitment"IEEE Transactions on Power Systems, Vol.7, $\mathrm{n}^{\circ} 2$, pp 591-599, May.

Lee, F.N. (1991). "The Application of Unit Commitment Utilization Factor (CUF) to Thermal Unit Commitment" IEEE Transactions on Power Systems, Vol.6, $\mathrm{n}^{\circ} 2$, pp.691-698, May.

Lowery, P.G. (1966). "Generating Unit Commitment by Dynamic Programming" IEEE Transactions on Power Systems, Vol. PAS-85, pp.422-426, May.

Lustig. I.J., Marsten, R.E. and Shanno, D.F. (1989). “Computational Experience with a Primal-Dual Interior Point Method for Linear Programming", Linear Algebra and Its Applications 1: 20-83.

Mantawy, A.H., Abdel-Magid, Y.L. and Selim, S.Z. (1998). "A simulated annealing algorithm for unit commitment," IEEE Trans. Power Syst., vol. 13, pp. 197-204, Feb.
Narayana Prasad Padhy (2004). "Unit Commitment - A Bibliographical Survey” IEEE Trans. Power Syst., vol. 19, no2, pp. 1197-1205, May.

Oliveira, E.J., Junior, I.C.S., Pereira, J.L.R.,Garcia, P.A.N and Sandoval C.J. (2005). "Transmission System Expansion Planning Using a Sigmoid Function to Handle Integer Investment Variables", IEEE Trans. on Power Systems, Vol. 20, $\mathrm{N}^{o}$ 3,Aug, pp. 1616-1621.

Ongsakul, W. and Petcharaks, N. (2004). "Unit Commitment by Enhanced Adaptive Lagrangian Relaxation" IEEE Trans. Power Syst., vol.19, ${ }^{\circ}$. 1, pp.620-628.

Pang, C.K., Sheble, G.B. and Albu, F. (1981). "Evaluation of Dynamic Programming Based Methods and Multiple Area Representation for Thermal Unit Commitment" IEEE Trans. Power App. Syst., Vol. PAS-100, pp.12121218, March.

Quintana, V. H., Torres, G. L. and Medina, J.P. (2000). "Interior-Point Methods and Their Applications to Power Systems: A Classification of Publications and Software Codes", IEEE Trans. Power Syst., vol. 15, no. 1, pp. 170—176, February.

Rajan, A.,Mohan. C.C. and Manivannan, M.R. (2002). "Neural based tabu search method for solving unit commitment problem," in Proc. 5th Int. Conf. Power Syst. Manage. Contr, pp. 180-185.

Sasaki, H., Watanabe, M. and Yokoyama, R. (1992). "A solution method of unit commitment by artificial neural networks," IEEE Trans. Power Syst., vol. 7, pp. 974981, Aug.

Senjyu, T., Yamashiro, H., Shimabukuro, K., Uezato, K. and Funabashi, T. (2003). "Fast Solution Technique for Large Scale Unit Commitment Problem Using Genetic Algorithm" IEE Proc.Gener.Transm. Distrib. vol.150, $\mathrm{n}^{\circ} .6$, November.

Senjyu, T., Yamashiro, H., Shimabukuro, K., Uezato, K. and Funabashi, T. (2002). "A Unit Commitment Problem by Using Genetic Algorithm Based on Characteristic Classification" IEEE/Power Eng. Soc. Winter Meet.,. vol.1, pp.58-63.

Senjyu, T., Shimabukuro, K., Uezato, K. and Funabashi, T. (2003).“A Fast Technique for Unit Commitment Problem by Extended Priority List" IEEE Transactions on Power Systems, Vol.18, no 2, pp.882-888, May.

Simopoulos, D.N., Kavatza, S.D. and Vournas, C.D. (2006). "Unit commitment by an enhanced simulated annealing algorithm" Power Systems, IEEE Transactionsn Volume 21, Issue 1, Feb, Page(s):68 - 76. 
Srinivasan, D and Chazelas, J. (2004). "A Priority List Based Evolutionary Algorithm to Solve Large Scale Unit Commitment Problem", International Conference on Power System Technology-Powercon 2004, Singapore, 21-24 November.

Sum, T. and Ongsakul, W. (2003). "Ant Colony Search Algorithm for Unit Commitment", Industrial Technology, IEEE International Conference Volume 1, 10-12 Dec, Page(s):72 - 77.

Swarup, K.S. and Yamashiro, S. (2002). "Unit commitment solution methodology using genetic algorithm," IEEE Trans. Power Syst., vol. 17, pp. 87-91, Feb.

Valenzuela, J. and Smith, A.E. (1999)."A Seeded Memetic Algorithm for Large Unit Commitment Problems" Journal of Heuristics, September.

Viana, A., Sousa, J.P. and Matos, M.A. (2003). "GRASP with Constraint Oriented Neighborhoods: an application to the Unit Commitment Problem" The Fifth Metaheuristics International Conference, Kyoto, Japan, August 25-28.

Wright, S.J.(1997) "Primal-Dual Interior Point Methods" Editora Siam.

Yang, H.T., Yang, P.C. and Huang, C.L. (1996). "Evolutionary programming based economic dispatch for units with non smooth fuel cost functions," IEEE Trans. Power Syst., vol. 11, pp. 112-118, Feb.

Zhuang, F. and Galiana, F.D. (1988). "Toward a more rigorous and practical unit commitment by Lagrangian Relaxation," IEEE Trans. Power Syst., vol.3, pp. 763-773, May.

\section{APÊNDICE A}

O problema de otimização pode ser formulado como:

$$
\begin{aligned}
& \operatorname{Min} f(x) \\
& \operatorname{s.a} \\
& h(x)=0 \\
& l \leq x \leq u
\end{aligned}
$$

onde:

$\begin{array}{llr}f(x) & \text { Função objetivo } & \text { A.2 } \\ h(x) & \text { Restrições de igualdade } & \text { A.3 } \\ l, u & \text { Limites inferiores e superiores das } & \text { A.4 } \\ & \text { variáveis } & \\ x & \text { Variáveis } & \text { A.5 } \\ \lambda, \pi & \text { Multiplicadores de Lagrange } & \text { A.6 }\end{array}$

Transformando as restrições de desigualdade em restrições de igualdade através da inclusão de variáveis de folga $(s l, s u)$, o problema (A.1) resulta no equivalente a:

$$
\begin{array}{ll}
\operatorname{Min} f(x) & \\
\operatorname{s.a} & \\
h(x)=0 & (\lambda) \\
x-s l=l & \left(\pi_{l}\right) \\
x+s u=u & \left(\pi_{u}\right) \\
s l, s u \geq 0 &
\end{array}
$$

Com o objetivo de transformar o problema de otimização sujeito apenas a restrições de igualdade (A.7) em um problema sem restrições, utiliza-se a função Lagrangeana $(L)$. Esta função é originada através de uma combinação linear entre as restrições (A.7). Os coeficientes desta combinação são denominados de multiplicadores de Lagrange $(\lambda, \pi)$. Também chamados de variáveis duais, os multiplicadores estão associados a cada restrição do problema de otimização. Assim, a função Lagrangeana referente ao problema (A.7) é dada por:

$$
L=f(x)-\lambda \cdot h(x)-\pi_{l}(x-s l-l)-\pi_{u}(x+s u-u)
$$

Com a função Lagrangeana obtida (A.8), parte-se para resolução da equação através das condições de otimalidade de KKT (Wright,1997), ou seja, deriva-se a equação Lagrangeana em relação as suas variáveis $(x, \lambda, \pi$,$) e iguala-se a zero.$ A seguir, o conjunto de equações é resolvido pelo método de Newton e com isso, têm-se os valores das variáveis $(x)$ e dos multiplicadores de Lagrange $(\lambda, \pi)$.

Os multiplicadores de Lagrange permitem interpretações econômicas importantes, pois traduzem a sensibilidade da função objetivo em relação às variações incrementais dos recursos das restrições.

\section{APÊNDICE B}

Os dados referentes ao sistema base com 10 unidades geradoras são apresentados nas tabelas B1 e B2. 


\begin{tabular}{|c|c|c|c|c|c|}
\hline & UTE 1 & UTE 2 & UTE 3 & UTE 4 & UTE 5 \\
\hline$P_{i}^{\max }$ & 455 & 455 & 130 & 130 & 162 \\
\hline$P_{i}^{\min }$ & 150 & 150 & 20 & 20 & 25 \\
\hline$a_{i}$ & 1000 & 970 & 700 & 680 & 450 \\
\hline$b_{i}$ & 16,19 & 17,26 & 16,60 & 16,50 & 19,70 \\
\hline$c_{i}$ & 0,00048 & 0,00031 & 0,002 & 0,00211 & 0,00398 \\
\hline$T_{i}^{\text {on }}$ & 8 & 8 & 5 & 5 & 6 \\
\hline$T_{i}^{\text {off }}$ & 8 & 8 & 5 & 5 & 6 \\
\hline$h c_{i}$ & 4500 & 5000 & 550 & 560 & 900 \\
\hline$c c_{i}$ & 9000 & 10000 & 1100 & 1120 & 1800 \\
\hline$c s h_{i}$ & 5 & 5 & 4 & 4 & 4 \\
\hline$C I_{i}$ & +8 & +8 & -5 & -5 & -6 \\
\hline
\end{tabular}

\begin{tabular}{|c|c|c|c|c|c|}
\hline & UTE 6 & UTE 7 & UTE 8 & UTE 9 & UTE 10 \\
\hline$P_{i}^{\max }$ & 80 & 85 & 55 & 55 & 55 \\
\hline$P_{i}^{\min }$ & 20 & 25 & 10 & 10 & 10 \\
\hline$a_{i}$ & 370 & 480 & 660 & 665 & 670 \\
\hline$b_{i}$ & 22,26 & 27,74 & 25,92 & 27,27 & 27,79 \\
\hline$c_{i}$ & 0,00712 & 0,00079 & 0,00413 & 0,00222 & 0,00173 \\
\hline$T_{i}^{o n}$ & 3 & 3 & 1 & 1 & 1 \\
\hline$T_{i}^{o f f}$ & 3 & 3 & 1 & 1 & 1 \\
\hline$h c_{i}$ & 170 & 260 & 30 & 30 & 30 \\
\hline$c c_{i}$ & 340 & 520 & 60 & 60 & 60 \\
\hline$c s h_{i}$ & 2 & 2 & 0 & 0 & 0 \\
\hline$C I_{i}$ & -3 & -3 & -1 & -1 & -1 \\
\hline
\end{tabular}

\begin{tabular}{|c|c|c|c|c|c|}
\hline$t$ & $D(t)$ & $t$ & $D(t)$ & $t$ & $D(t)$ \\
\hline 1 & 700 & 9 & 1300 & 17 & 1000 \\
\hline 2 & 750 & 10 & 1400 & 18 & 1100 \\
\hline 3 & 850 & 11 & 1450 & 19 & 1200 \\
\hline 4 & 950 & 12 & 1500 & 20 & 1400 \\
\hline 5 & 1000 & 13 & 1400 & 21 & 1300 \\
\hline 6 & 1100 & 14 & 1300 & 22 & 1100 \\
\hline 7 & 1150 & 15 & 1200 & 23 & 900 \\
\hline 8 & 1200 & 16 & 1050 & 24 & 800 \\
\hline
\end{tabular}

Tabela B2- Demanda -Sistema 10 Unidades.

Tabela B1: Características UTE‘s -Sistema 10 Unidades. 